INRA Prod. Anim., 1997, 10 (4), 275-285
C. Mignolet, M. Benoît, D. Saintôt

INRA Station de Recherche SAD, Domaine du Joly, BP 29, 88501 Mirecourt Cedex

\section{Systèmes d'élevage et risque de pollution azotée. Construction d'un indicateur de risque et application dans la plaine des Vosges}

Depuis deux décennies, un nombre croissant de sources souterraines d'eau potable présente une augmentation des teneurs en nitrates, qui dépassent la norme européenne de $50 \mathrm{mg} / \mathrm{l}$ et inquiètent les collectivités locales concernées. Dans les zones rurales, les activités agricoles sont la principale cause de pollution par les nitrates. La mise au point d'un indicateur de risque de pollution selon les types de système de production et sa spatialisation sur l'ensemble d'une région permet d’identifier les systèmes à risque et les zones agricoles sensibles.

Depuis une vingtaine d'années, la responsabilité des pratiques agricoles modernes dans l'accroissement des niveaux de pollution des ressources en eau est prouvée et admise (Hénin et al 1980). Dans le cas des activités d'élevage de ruminants, l'impact des pratiques agricoles sur la qualité des ressources en eau revêt deux formes différentes (Coppenet 1975, Pflimlin et Madeline 1995) :

\footnotetext{
Résumé

Une méthode d'évaluation et de localisation des risques de pollution nitrique diffuse des eaux souterraines, liés à la diversité des systèmes d'élevage de ruminants et à leurs assolements caractéristiques, est mise au point et appliquée sur les 2211 exploitations présentes en 1993 dans la plaine des Vosges. Cette méthode associe des mesures des lixiviations nitriques réelles sous des parcelles agricoles, une typologie des exploitations d'élevage qui contient des informations sur les assolements et un système d'information géographique qui permet de localiser les informations au sein d'unités territoriales de gestion des ressources en eau. Les exploitations combinant des surfaces importantes en grandes cultures et un système fourrager à base de maïs génèrent actuellement les plus forts risques de pollution par les nitrates, alors que les exploitations spécialisées dans les productions de lait et/ou de viande à base d'herbe apparaissent les moins polluantes. La localisation des types d'exploitation permet d'identifier des zones géographiques exposées à un fort risque de pollution d'origine agricole, caractérisées par une combinaison des types d'exploitation d'élevage les plus modernisés et intensifs de la région.
}

- une pollution directe - organique, microbienne ou phosphatée - des eaux de surface, dont la maîtrise passe par la mise en conformité des bâtiments d'élevage et des capacités de stockage des déjections animales et des fourrages, ainsi que par le respect des recommandations sur les modalités et les périodes d'épandage des fumiers et lisiers ;

- une pollution diffuse, spécifiquement liée aux apports d'azote et de pesticides sur les cultures et les prairies, ceux-ci atteignant les eaux souterraines par infiltration après épandage. Cette pollution diffuse dépend essentiellement de deux paramètres : les assolements (Machet et Mary 1990, Gaury 1992a et $1992 b)$, qui révèlent une plus ou moins grande intensification fourragère, et les pratiques culturales (itinéraires techniques), notamment les pratiques d'épandage des déjections animales (Le Houérou 1993).

Afin de cibler au mieux les mesures de protection des ressources en eau qui sont non seulement définies aux niveaux national et communautaire mais également à des niveaux plus locaux (région, bassin), il apparaît nécessaire d'identifier les systèmes d'exploitation qui présentent un risque de pollution et de localiser les zones géographiques où ce risque de pollution existe. Cet article présente une méthode d'évaluation et de localisa- 
tion des risques de pollution nitrique diffuse des eaux souterraines, liés à la diversité des systèmes d'élevage de ruminants et aux assolements qui les caractérisent (Mignolet et al 1996). Cette méthode est appliquée à l'échelle de la plaine des Vosges, région de polycultureélevage du nord-est de la France qui s'étend sur 380000 ha. Cette région, à dominante herbagère, connaît des problèmes importants de pollution des eaux souterraines par les nitrates, car elle présente une hydrogéologie particulière qui fait que de nombreuses sources d'eau potable sont situées en contrebas de plateaux karstiques sablo-limoneux, sièges de l'intensification fourragère (KüngBenoît 1992). Ces sources ont fréquemment des teneurs en nitrates supérieures au seuil de potabilité de $50 \mathrm{mg} / \mathrm{l}$.

La méthode proposée comprend deux phases distinctes : d'une part une formalisation de la diversité des systèmes d'élevage de la région d'étude par construction d'une typologie d'exploitation et localisation des types, et d'autre part une quantification des pertes en nitrates des différents systèmes de culture mis en place par les exploitations d'élevage. Les résultats de ces deux phases sont ensuite combinés pour construire un indicateur de risque de pollution nitrique par type d'exploitation. Ils sont présentés à l'échelle des communes et à l'échelle de nouvelles unités territoriales de gestion des ressources en eau : les SAGE (Schéma d'Aménagement et de Gestion des Eaux).

$\mathrm{Au}$ total, quatre échelles spatiales sont utilisées : la parcelle culturale, l'exploitation agricole, la commune et le SAGE. Mise à part la parcelle culturale choisie parce qu'elle permet d'associer de façon sûre un système de culture à une qualité d'eau, les trois autres échelles ont été choisies parce que ce sont des niveaux d'organisation qui correspondent à des lieux d'action : l'exploitation agricole est dirigée par un ou plusieurs chefs d'exploitation, la commune est gérée par un Conseil municipal, et le SAGE est sous la responsabilité d'un Comité Local de l'Eau. Construire des indicateurs de risque de pollution à ces niveaux peut donc orienter et aider la prise de décision et l'application de mesures visant à protéger la qualité des ressources en eau.

\section{1 / Diversité des systèmes d'élevage}

\section{1 / Les types d'exploitation}

L’objectif étant de relier systèmes d'élevage et pollution nitrique de l'eau, nous cherchons à caractériser les exploitations d'élevage par leur logique de fonctionnement, qui dépend en partie des niveaux de modernisation et d'intensification des systèmes techniques. Une typologie d'exploitation, construite par la Chambre d'Agriculture des Vosges selon la méthode à dires d'experts décrite par Perrot
(1990), a donc été utilisée. Cette méthode identifie des " pôles d'agrégation » qui résument, par une combinaison d'indicateurs discriminants, les caractéristiques des différents groupes d'exploitations définis par les experts. L'ensemble des exploitations se rapprochant le plus d'un pôle est appelé un type.

Douze types d'exploitation d'élevage (neuf types laitiers et trois types viande) ont ainsi été définis à partir des 2211 exploitations présentes en 1993 dans la zone étudiée (figure 1). Un cinquième de ces exploitations n'a pu être rattaché à aucun type ; c'étaient soit des exploitations de grandes cultures spécialisées, soit des exploitations d'élevage qui suivaient une logique de fonctionnement particulière, voire marginale (par exemple les exploitations biologiques). L'analyse de ces exploitations pourrait conduire à modifier les types existants ou à en créer de nouveaux si le résidu des exploitations non typées contient un ou plusieurs groupes d'exploitations caractéristiques d'une logique de fonctionnement jusque là non prise en compte par la typologie (en particulier, la création d'un type caractérisant les exploitations céréalières spécialisées nous semblerait pertinente, notamment pour la partie nord-ouest du département). C'est un des gros avantages de la typologie par agrégation que de pouvoir être aisément actualisée au fil de l'évolution de l'agriculture (Perrot et al 1995). Dans le présent travail, les indicateurs de risque de pollution ne tiennent pas compte des exploitations non typées, pour lesquelles nous n'avions accès à aucun renseignement.

Un assolement spécifique, caractéristique d'une logique technique correspond à chaque type d'exploitation (tableau 1). Les types intitulés Société, Lait-Céréales et CéréalesViande présentent les surfaces en céréales et en colza les plus importantes (près de la moitié de la SAU moyenne), ainsi qu'une part élevée de la surface en maïs fourrage. Le type Lait Modernisé Maïs Viande possède des surfaces en cultures de vente beaucoup moins importantes (moins de $20 \%$ de la SAU), mais la proportion de maïs y est supérieure à celle des types précédents (17\% de la SAU, ce qui représente, comme pour les types précédents, un peu moins du quart de la SFP). Les autres types laitiers sont avant tout caractérisés par les surfaces toujours en herbe, qui représentent environ $70 \%$ de la SAU pour les types Lait Non Modernisé, Lait à Modernisation limitée Maïs et Lait Modernisé Maïs limité Viande, jusqu'à plus de $80 \%$ de la SAU pour les types Lait Ensilage d'Herbe, Lait-FoinRegain et Lait à Modernisation limitée Herbe, le reste étant constitué de céréales et d'autres cultures fourragères. Enfin, les types herbagers de production de viande, Herbager bovin et Herbager ovin, présentent des assolements presque entièrement en herbe (respectivement $92 \%$ et $96 \%$ de STH dans la SAU).

Cette typologie d'exploitation a une vocation générale de description de l'agriculture régionale, et est utilisée dans ce but par l'ensemble des organismes professionnels agri- 
Figure 1. Représentation graphique des douze types d'exploitation.

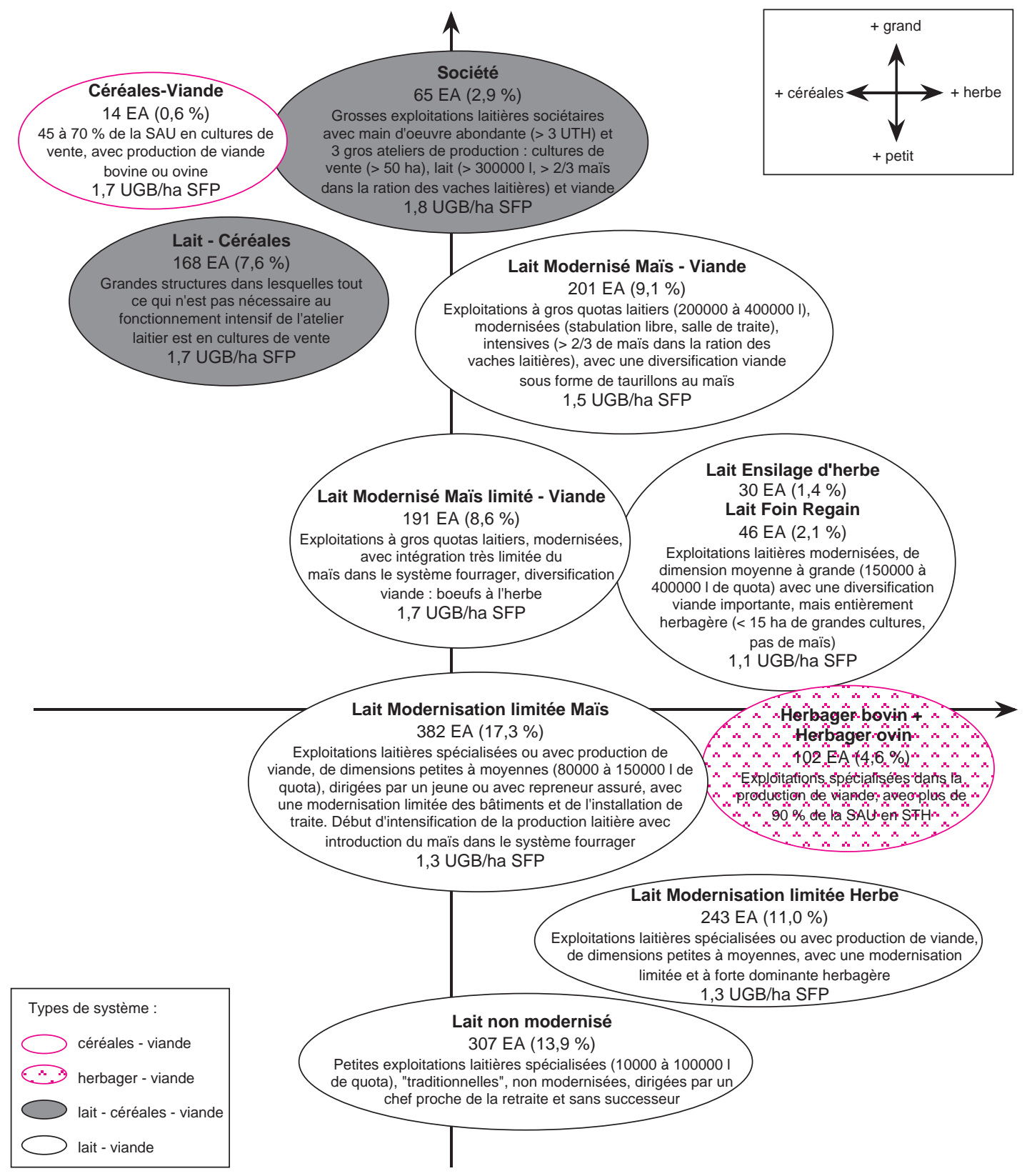

Les 2211

exploitations de la plaine des Vosges ont été réparties en 12 types selon leurs caractéristiques. A chaque type correspond un assolement spécifique.

Tableau 1. Assolement moyen par type d'exploitation (en ha).

\begin{tabular}{|l|r|r|r|r|r|r|r|r|r|r|r|r|}
\hline & $\mathrm{S}^{(1)}$ & \multicolumn{1}{|c|}{ LC } & LMMV & LMMlV & LEH & LFR & LMIM & LMlH & LNM & HB & HO & CV \\
\hline Céréales & 68,4 & 41,6 & 13,8 & 10,5 & 4,7 & 11,0 & 9,8 & 5,8 & 5,7 & 5,9 & 1,4 & 42,5 \\
$\quad$ Blé & 38,5 & 22,7 & 6,8 & 5,1 & 2,4 & 5,1 & 4,8 & 2,9 & 3,1 & 3,1 & 0,8 & 23,6 \\
$\quad$ Orge & 26,6 & 16,6 & 6,4 & 5,0 & 2,3 & 5,9 & 4,7 & 2,9 & 2,6 & 2,2 & 0,6 & 15,7 \\
$\quad$ Maïs grain & 3,3 & 2,3 & 0,6 & 0,4 & 0,0 & 0,0 & 0,3 & 0,0 & 0,0 & 0,6 & 0,0 & 3,2 \\
Colza & 20,3 & 12,5 & 1,0 & 0,8 & 0,0 & 0,9 & 0,7 & 0,1 & 0,6 & 0,3 & 0,0 & 13,5 \\
Maïs fourrage & 23,5 & 13,6 & 14,5 & 5,8 & 0,0 & 0,0 & 4,2 & 0,0 & 0,0 & 3,4 & 0,0 & 5,0 \\
Surface toujours & & & & & & & & & & & & \\
en herbe & 73,3 & 36,7 & 48,9 & 56,7 & 50,5 & 61,3 & 34,6 & 36,7 & 19,8 & 112,3 & 51,4 & 47,0 \\
Autres cultures & & & & & & & & & & & & \\
fourragères & 8,0 & 6,0 & 4,9 & 4,0 & 6,5 & 2,6 & 2,3 & 1,9 & 1,2 & 1,0 & 0,5 & 2,8 \\
Surface agricole & & & & & & & & & & & & \\
utile & 193,5 & 110,4 & 83,1 & 77,8 & 61,7 & 75,8 & 51,6 & 44,5 & 27,3 & 122,9 & 53,3 & 110,8 \\
\hline
\end{tabular}

(1) Ces intitulés correspondent aux initiales des noms des types (voir texte). 
coles des Vosges et de la Lorraine (Institut de l'Elevage, Chambres d'Agriculture régionale et départementale, Centre de Gestion). Nous aurions pu choisir d'élaborer une autre typologie qui classe les exploitations en fonction de caractéristiques spécifiquement liées à la pollution nitrique (type de bâtiments d'élevage, capacité de stockage des fumiers et lisiers, quantité de déjections animales par rapport à la surface d'épandage, détail des fertilisations organiques et minérales ...). Ce choix ne nous a pas paru judicieux pour deux raisons principales :

- une telle typologie n'aurait pas pu être utilisée sur une grande échelle, car il n'existe pas de banque de données régionale comportant ce type d'information et il nous aurait été impossible de mettre en place et d'assumer un protocole d'enquêtes sur plusieurs centaines d'exploitations ;

- la typologie à dires d'experts est en partie basée sur des indicateurs qui discriminent les exploitations en fonction des niveaux d'intensification de leur système technique : nombre d'hectares de maïs, pratique de l'ensilage d'herbe, ration hivernale des vaches laitières, type de mâle engraissé (taurillon au maïs ou bœuf à l'herbe). Ces indicateurs ne sont pas directement liés aux pertes en nitrates, mais ils les renseignent indirectement car ils sont liés aux assolements.

\section{2 / Localisation des types d'exploitation}

Le principe retenu est de regrouper les communes, qui constituent le plus petit maillage statistique usuel, qui présentent des profils agricoles semblables ou proches, un profil agricole communal étant défini comme la combinaison des types d'exploitation dont le siège est dans la commune. La typologie de communes a été construite par des méthodes d'analyses de données multivariées (analyse factorielle des correspondances appliquée au tableau de contingence des nombres d'exploitations par type et par commune, puis classification ascendante hiérarchique selon la méthode du minimum de variance de Ward sur les trois premiers facteurs de l'analyse factorielle, Mignolet 1996).

Cette méthode de localisation des types d'exploitation est soumise à l'hypothèse d'inclusion des parcellaires d'exploitation à l'intérieur des limites de la commune de leur siège. Or, nous pouvons a priori penser que nombre d'exploitations des types Société, LaitCéréales, Lait Modernisé Maïs Viande et Céréales-Viande, qui se placent souvent sur des trajectoires d'agrandissement, étendent leur parcellaire sur plusieurs finages communaux. Ce biais, qui paraît actuellement acceptable dans la zone d'étude retenue, risque de s'amplifier si la baisse de la démographie agricole se poursuit.

La figure 2 illustre les dix combinaisons de profils agricoles existant sur les 365 communes de la plaine des Vosges. Elle montre que la répartition des types d'exploitation n'y est pas uniforme. Certaines zones caractérisées par des combinaisons de systèmes d'élevage intensifs, semblent plus exposées que d'autres à des problèmes de pollution nitrique. Ainsi, les types Société, LaitCéréales, Lait Modernisé Maïs Viande et Céréales-Viande apparaissent préférentiellement localisés autour des centres urbains du département (plateaux de Neufchâteau, triangle Vittel-Mirecourt-Escles, Rambervillers et Epinal) et le long des grandes voies de communications routières (axe Mirecourt-Epinal notamment). A l'inverse, les types herbagers plus extensifs (lait ou viande) se retrouvent dans le Châtenois et dans le sud de la plaine des Vosges. La région de Lamarche, au sudouest, située sur des terrains lourds, est dominée par des types laitiers herbagers modernisés à non modernisés, alors que dans le secteur de Xertigny, au sud-est, quelques communes situées sur des terrains du grès vosgien (terrains faciles à travailler qui autorisent la culture du maïs) sont caractérisées par des types laitiers avec introduction limitée du maïs dans les systèmes fourragers.

\section{2 / Risques de pollution des eaux souterraines liés au type d'exploitation}

\section{1 / Quantification des lixiviations nitriques}

La pollution nitrique des eaux souterraines due aux cultures assolées est évaluée grâce à la mise en place d'un dispositif de mesure sous parcelles agricoles. Depuis 1989, quarante-trois sites de sept bougies poreuses (cf encadré), enterrés à un mètre de profondeur sous un échantillon de parcelles culturales représentatives et répartis dans une gamme variée de types de sols (des argiles de la Lettenkohle aux petites terres à cailloux sur calcaires à cératites), permettent de suivre régulièrement les teneurs en nitrates de l'eau drainante. Les données météorologiques nécessaires au calcul de la lame d'eau drainante, et les qualités d'eau drainée sous chaque parcelle sont relevées tous les quinze jours. La lame d'eau drainante $\left(D_{c}\right)$ sous chaque parcelle est donnée par la formule suivante (Gaury 1992a) :

$\mathrm{D}_{\mathrm{c}}=\mathrm{P}$ - k. ETP - RU

avec $\mathrm{P}=$ pluviométrie (en $\mathrm{mm}$ )

$\mathrm{k}=$ coefficient de Robelin

ETP de Penmann (en mm)

$\mathrm{RU}=$ réserve utile du sol (en $\mathrm{mm}$ )

La synthèse des valeurs des lixiviations nitriques par parcelle est réalisée sous l'hypothèse de l'affectation des eaux à la culture d'une parcelle durant la période qui sépare sa fertilisation azotée et la fertilisation azotée de la culture suivante. Pour chaque site, la concentration moyenne des nitrates $\left(\left[\mathrm{NO}_{3}\right]_{\text {moy }}\right)$ associée à la culture assolée est calculée par double cumul du flux d'eau et du flux d'azote 
Figure 2. Typologie des communes de la Plaine des Vosges.

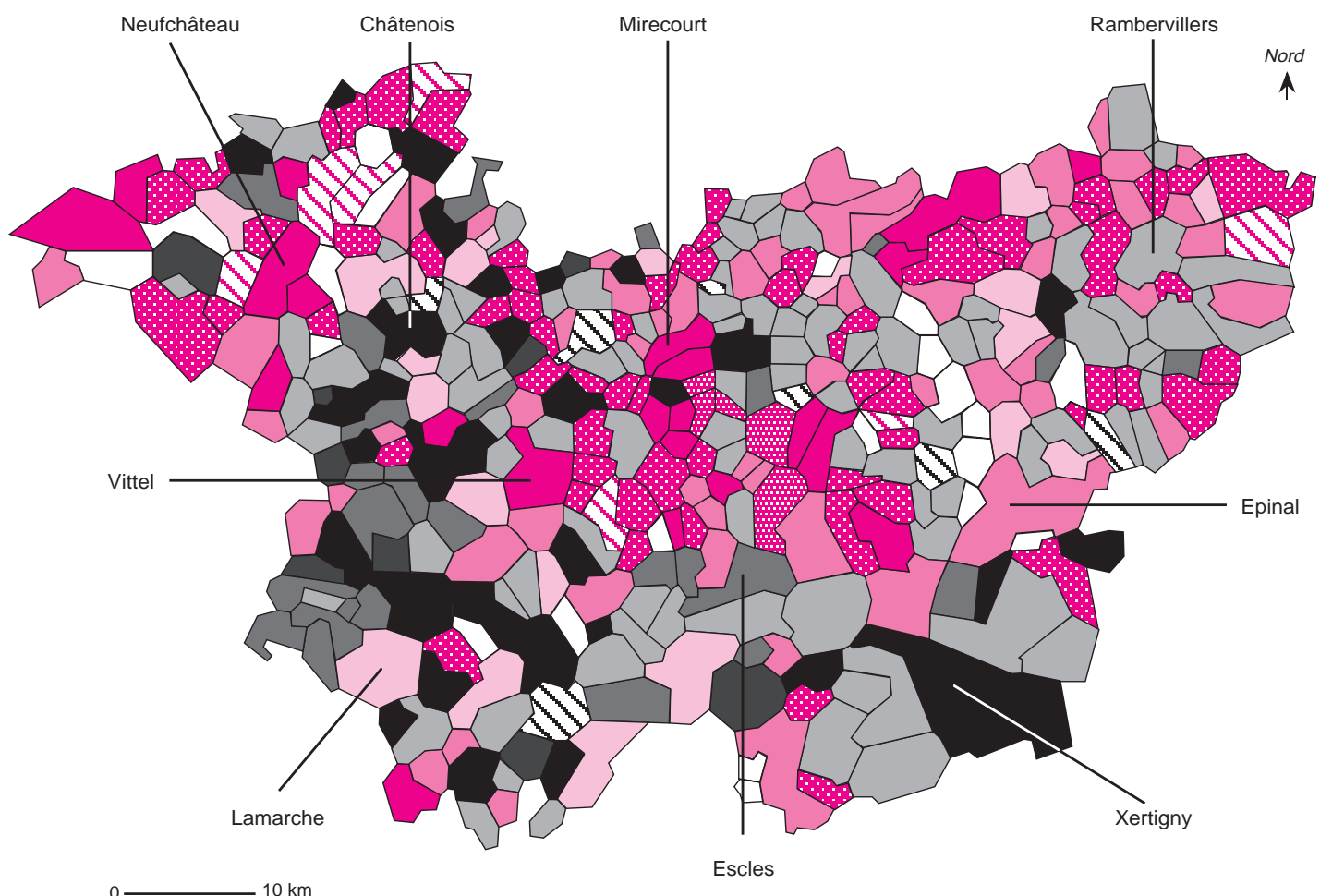

Société + Lait-Céréales + Lait modernisé Maïs Viande

Lait-Céréales + Lait modernisé Maïs Viande

Société + Lait-Céréales + Lait à modernisation limitée Maïs

Lait modernisé Maïs Viande + Lait à modernisation limitée Maïs et Herbe

Lait modernisé Maïs limité Viande + Lait à modernisation limitée Maïs et Herbe + Lait non modernisé

$\square$ Types laitiers herbagers modernisés à non modernisés + Herbager bovin

Types laitiers herbagers modernisés ou à modernisation limitée + Herbager bovin + Herbager ovin

Lait à modernisation limitée Herbe + Lait non modernisé

Herbager ovin

Céréales-Viande

$\left(\mathrm{LNO}_{3}\right)$ mesurés entre deux printemps successifs, selon la droite de régression suivante (Benoît et al 1995a) :

$\mathrm{LNO}_{3}=\left(\left[\mathrm{NO}_{3}\right]_{\text {moy }} \times \mathrm{D}_{\mathrm{c}}\right)+$ constante

Le tableau 2 présente les moyennes des valeurs obtenues sur les quarante-trois sites équipés de bougies poreuses entre 1989 et 1995. Les écarts-types de ces moyennes sont parfois très importants en raison des paramètres suivants :

- le type de sol et le climat (pluviosité et température) qui jouent sur l'ampleur du lessivage des nitrates : la minéralisation de l'azote dans le sol pendant la période automnale est plus importante en année chaude et sèche qu'en année froide et humide (Duthil 1973). De même, le risque de lessivage est accru dans un sol filtrant et peu profond tel un sol sableux superficiel (à faible réserve utile) par rapport à un sol limoneux et a fortiori à un sol argileux profond (à réserve utile élevée, Hénin et al 1980) ;

- les paramètres techniques : les pertes de nitrates dépendent en grande partie des quantités, de la forme et des périodes d'apport de l'azote aux cultures, mais aussi du travail
La répartition géographique des 12 types n'est pas uniforme et la prédominance de systèmes intensifs dans certaines communes les exposent à un risque plus élevé de pollution azotée.

\section{Fonctionnement des sites à bougies poreuses en situation agricole}

Le suivi de la cinétique des pertes de la solution du sol vers les nappes phréatiques nécessite de disposer de capteurs en place, qui permettent de récupérer pour analyse des échantillons de solution du sol. La Station de Mirecourt a ainsi choisi d'installer un dispositif in situ qui n'entrave en rien l'exécution normale des pratiques agricoles (travail du sol, pâturage ...). Ce dispositif est constitué de sites à bougies poreuses enterrées horizontalement à un mètre de profondeur et raccordées à une station de prélèvement en bordure de parcelles, qui permet la récupération des solutions sans avoir à pénétrer dans ces parcelles (Barlier 1991). Les prélèvements d'eau accumulée dans les bougies poreuses sont effectués 48 heures après avoir réalisé un vide d'air, avec une périodicité de 15 jours. Un site à bougies poreuses est implanté sous chaque parcelle cultivée. Un à trois sites sont implantés sous les parcelles pâturées (Fiorelli et al 1995). 
du sol et du mode de gestion des résidus de culture. Les rotations culturales et l'implantation de cultures intermédiaires ont également une influence. Pour les surfaces pâturées, les pertes de nitrates sont principalement liées au chargement et au nombre de jours de pâturage. Les itinéraires techniques adoptés par les agriculteurs pour chaque culture ou chaque type de prairie sont une des variables explicatives principales du lessivage. Or, ils paraissent extrêmement variés, non seulement entre les exploitations mais également dans une même exploitation, où ils peuvent varier d'une année sur l'autre en fonction de l'évolution du contexte économique et réglementaire.

La part de chacun de ces paramètres, et notamment celle des pratiques agricoles, dans la valeur des écarts-types n'est actuellement pas quantifiable. Cent quatre-vingt douze observations sont disponibles depuis la mise en place du dispositif sous les parcelles agricoles, ce qui représente un nombre encore faible d'observations par utilisation du sol, et donc un nombre encore plus faible d'itinéraires techniques différents.

Cette étude ne prend en compte que les valeurs moyennes de lixiviation nitrique par occupation du sol, sans intégrer leur variabilité. En effet, nous considérons que les écarts liés aux couverts végétaux "sur-déterminent " les effets liés au milieu, au climat et aux pratiques agricoles.

Dans le cas des exploitations d'élevage avec peu de cultures de vente, le facteur principal qui joue sur la lixiviation nitrique est le choix du système fourrager hivernal (Benoît et al 1995b) : dans la plaine des Vosges, il est constitué soit par de l'herbe (sous forme de foin ou d'ensilage) soit par du maïs fourrager. Un système fourrager hivernal à base d'herbe induit un faible niveau de lixiviations nitriques (moins de $25 \mathrm{mg} / \mathrm{l}$ ). Pour un système fourrager à base de maïs, ce niveau s'accroît en moyenne jusqu'à 48 mg/l (tableau 2).

Les cultures de vente (colza, blé, orge ...), dans le cas des exploitations d'élevage qui y consacrent une part importante de leur SAU, génèrent le plus grand risque de pollution par les nitrates. Les lixiviations moyennes dépas- sent en effet largement le seuil de potabilité de $50 \mathrm{mg} / \mathrm{l}$ pour les céréales d'hiver, jusqu'à atteindre près de $100 \mathrm{mg} / \mathrm{l}$ pour la culture de colza. Les exploitations d'élevage associant à la fois des surfaces importantes en grandes cultures et un système fourrager à base de maïs présentent donc les plus forts risques potentiels de pollution nitrique des eaux souterraines. Nous verrons ultérieurement que ces risques peuvent être nuancés en fonction de la localisation des exploitations par rapport aux bassins d'alimentation des sources d'eau potable.

\section{2 / Définition d'un indicateur de risque de pollution nitrique par type d'exploitation}

Pour chaque type d'exploitation, un indicateur de risque de pollution nitrique $\mathrm{I}_{\mathrm{T}}$ est défini en fonction des surfaces des différentes occupations du sol caractéristiques du type et des lixiviations moyennes des nitrates qui leur sont liées :

$\mathrm{I}_{\mathrm{T}}=\left(\sum \mathrm{S}_{\mathrm{i}} \times\left[\mathrm{NO}_{3}\right]_{\mathrm{i}}\right) / \mathrm{SAU}_{\mathrm{T}}$ avec : $\mathrm{S}_{\mathrm{i}}$ la surface de la culture $\mathrm{i}$

$\left[\mathrm{NO}_{3}\right]_{\mathrm{i}}$ la lixiviation moyenne des nitrates liée à la culture $\mathrm{i}$

$\mathrm{SAU}_{\mathrm{T}}$ la SAU moyenne du type

Cet indicateur simple permet d'associer à chaque type une valeur théorique de lixiviations et de hiérarchiser les types en fonction des risques de pollution nitrique qu'ils présentent. Ainsi les types Herbager ovin, Herbager bovin, Lait à Modernisation limitée Herbe, Lait-Foin-Regain et Lait Ensilage d'Herbe ont un indicateur de risque inférieur à $31 \mathrm{mg} / \mathrm{l}$ (figure 3). Ce sont des types d'exploitation laitiers ou viande spécialisés (sans grandes cultures) caractérisés par des systèmes fourragers herbagers. Les surfaces en herbe, en moyenne moins polluantes que les cultures quelle que soit leur utilisation, constituent la majeure partie de leur assolement.

A l'opposé, les indicateurs de risque des types Société, Lait-Céréales et CéréalesViande dépassent $45 \mathrm{mg} / \mathrm{l}$. Ces types représentent des exploitations de polyculture-élevage intensives et de grande dimension, dans

Tableau 2. Quantités (moyennes + écarts-types) de nitrates lixiviés et d'eau drainée selon l'utilisation du sol.

\begin{tabular}{|l|c|c|c|c|c|c|}
\hline & \multicolumn{3}{|c|}{1989 à 1995 } & \multicolumn{3}{c|}{1991 (année sèche) } \\
\cline { 2 - 7 } & $\begin{array}{c}\text { Nombre } \\
\text { d'observations }\end{array}$ & $\begin{array}{c}\text { Nitrates } \\
(\mathrm{mg} / \mathrm{l})\end{array}$ & $\begin{array}{c}\text { Eau drainée } \\
(\mathrm{mm} / \mathrm{an})\end{array}$ & $\begin{array}{c}\text { Nombre } \\
\text { d'observations }\end{array}$ & $\begin{array}{c}\text { Nitrates } \\
(\mathrm{mg} / \mathrm{l})\end{array}$ & $\begin{array}{c}\text { Eau drainée } \\
\text { (mm/an) }\end{array}$ \\
\hline Colza & 2 & $93 \pm 7$ & $403 \pm 35$ & & & \\
Blé d'hiver & 20 & $57 \pm 30$ & $480 \pm 119$ & & & \\
Orge d'hiver & 10 & $75 \pm 55$ & $326 \pm 165$ & 6 & $87 \pm 57$ & $227 \pm 38$ \\
Blé et Orge de printemps & 11 & $30 \pm 26$ & $484 \pm 125$ & 1 & 6 & 227 \\
Maïs & 55 & $48 \pm 31$ & $456 \pm 136$ & 6 & $78 \pm 30$ & $218 \pm 55$ \\
Pâture vaches laitières & 38 & $34 \pm 48$ & $407 \pm 141$ & 3 & $56 \pm 53$ & $218 \pm 40$ \\
Pâture autres bovins & 19 & $18 \pm 20$ & $365 \pm 158$ & 3 & $20 \pm 25$ & $130 \pm 78$ \\
Pré de fauche & 14 & $17 \pm 19$ & $424 \pm 133$ & 1 & 41 & 245 \\
Prairies temporaires & 14 & $30 \pm 48$ & $413 \pm 122$ & & & 166 \\
Luzerne & 9 & $23 \pm 27$ & $372 \pm 138$ & 1 & 8 & 166 \\
\hline
\end{tabular}


lesquelles l'alimentation des troupeaux est à base de maïs ensilage au moins pendant la période hivernale, et même parfois tout au long de l'année. Leur assolement est principalement constitué de céréales, de colza et de maïs, qui sont les occupations du sol actuellement les plus polluantes.

En position intermédiaire, les types Lait Modernisé Maïs Viande, Lait Modernisé Maïs limité Viande, Lait à Modernisation limitée Maïs et Lait Non Modernisé présentent un indicateur de risque compris entre 32 et $35 \mathrm{mg} / \mathrm{l}$. Avec peu de cultures de vente, c'est la présence du maïs dans le système fourrager qui explique ces valeurs supérieures à celles des types herbagers (mis à part pour le type Lait Non Modernisé).

Une limite au calcul de cet indicateur de pollution tient à la définition des assolements réalisée pour chaque type d'exploitation : l'information disponible dans la banque de données ne permet pas de les détailler plus finement.

Tout d'abord, les céréales d'hiver n'ont pu être distinguées des céréales de printemps. Or, les valeurs de lixiviations nitriques mesurées dans les sites à bougies poreuses varient de $57 \mathrm{mg} / \mathrm{l}$ et $75 \mathrm{mg} / \mathrm{l}$ en moyenne respectivement pour le blé et l'orge d'hiver à $30 \mathrm{mg} / \mathrm{l}$ en moyenne pour les céréales de printemps (tableau 2) qui reçoivent une fertilisation azotée beaucoup plus faible. Sachant que, dans les Vosges, les céréales de printemps représentent moins du tiers des récoltes en céréales totales, l'indicateur de risque a été calculé à partir des mesures réalisées sur céréales d'hiver, ce qui conduit à une légère surestimation des risques de pollution.

Ensuite, il s'est avéré impossible de différencier les surfaces toujours en herbe en fonction de leur mode d'utilisation. Or, celui-ci influe sur les lixiviations nitriques qui varient de $17 \mathrm{mg} / \mathrm{l}$ et $18 \mathrm{mg} / \mathrm{l}$ pour les prés de fauche et les parcs pâturés par les génisses et les bœufs respectivement, à $34 \mathrm{mg} / \mathrm{l}$ pour les parcs à vaches laitières, soit une variation des moyennes du simple au double. L'indicateur de risque a été calculé en utilisant la moyenne de ces trois valeurs, ce qui atténue les différences entre les types d'exploitation à gros troupeaux laitiers et ceux à petits troupeaux.

\section{3 / Spatialisation des risques de pollution nitrique à l'échelle d'unités régionales de gestion des ressources en eau}

\section{1 / Délimitation et rôle des SAGE}

Chacune des six Agences de Bassin françaises a mis en place en 1995 un Schéma Directeur d'Aménagement et de Gestion des Eaux (SDAGE) qui fixe les orientations fonda-
Figure 3. Indicateur de risque de pollution nitrique des douze types d'exploitation.

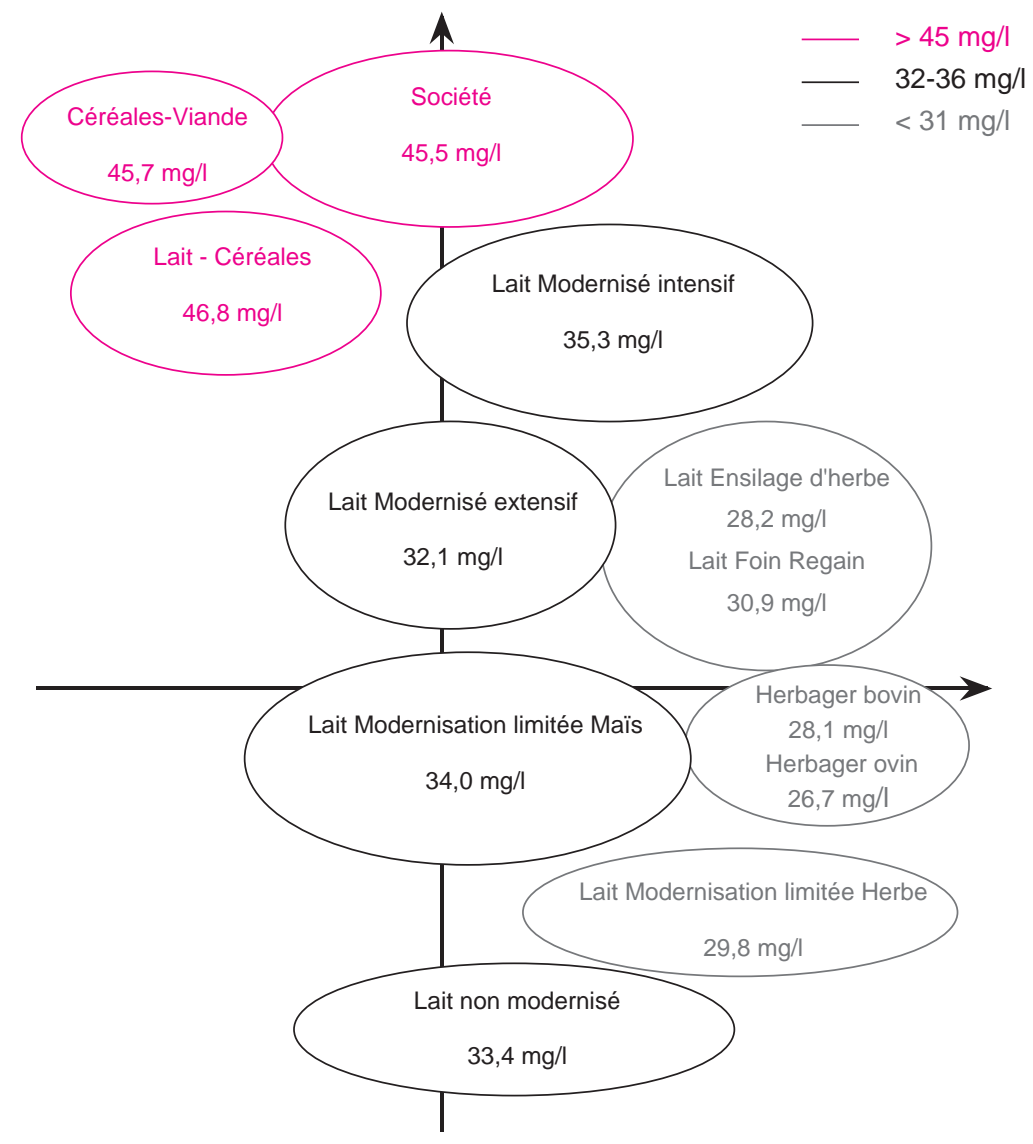

mentales d'une gestion équilibrée des ressources en eau et définit les objectifs de quantité et de qualité des eaux ainsi que les aménagements à réaliser pour les atteindre (Agence de l'Eau Rhin-Meuse 1995). Les dispositions de ce Schéma, dont les pollutions diffuses d'origine agricole constituent un volet, sont appliquées dans des SAGE qui sont des unités territoriales cohérentes du point de vue hydrographique et hydrogéologique. Pour chaque SAGE, un Comité Local de l'Eau est en charge de la gestion des ressources en eau et doit définir les objectifs et le suivi technique de toutes les activités localisées dans le SAGE.

Les maillages administratifs et hydrographiques n'étant pas superposables, la plaine des Vosges couvre une partie de quatre des trente-deux SAGE délimités dans le Bassin Rhin-Meuse : la Haute-Meuse, le Madon, la Haute-Moselle et la Mortagne (tableau 3). Pour la même raison, une commune peut appartenir à deux SAGE voisins. Pour simplifier, nous avons affecté chaque commune au SAGE qui couvre la plus grosse partie de son territoire.

\section{2 / Définition d'un indicateur de risque de pollution nitrique par SAGE}

Lindicateur de risque de pollution nitrique par SAGE, $\mathrm{I}_{\mathrm{S}}$, est une valeur théorique de
Pour chaque type d'exploitation, on définit un indicateur de risque de pollution azotée, qui intègre l'assolement et les quantités de nitrates drainés selon les utilisations du sol. 
Tableau 3. Caractéristiques et indicateur de risque $\left(l_{S}\right)$ de pollution nitrique (mg/l) par SAGE.

\begin{tabular}{|l|c|c|c|c|}
\hline & Haute-Meuse & Madon & Haute-Moselle & Mortagne \\
\hline $\begin{array}{l}\text { Surface (ha) } \\
\text { \% surface couvert }\end{array}$ & 149765 & 103621 & 101001 & 67852 \\
par la Plaine des Vosges & 67,2 & 59,6 & 71,0 & 57,6 \\
Nombre de communes & 102 & 91 & 65 & 36 \\
Is moyen 1989-1995 & 35,7 & 37,9 & 36,1 & 37,4 \\
- 1er quartile sur les communes & 31,4 & 33,4 & 32,9 & 33,9 \\
- médiane sur les communes & 33,1 & 35,2 & 34,0 & 36,2 \\
- 3e quartile sur les communes & 39,0 & 40,2 & 39,3 & 39,5 \\
Is 1991 (année sèche) & 49,2 & $\mathbf{5 1 , 8}$ & $\mathbf{4 9 , 8}$ & $\mathbf{5 0 , 9}$ \\
\hline
\end{tabular}

On peut de la même façon définir un indicateur de risque par SAGE, unité territoriale d'aménagement et de gestion des eaux. teneur en nitrates, fonction du nombre d'exploitations par type d'exploitation pour chaque SAGE, pondéré par la SAU moyenne de chaque type, et de l'indicateur de risque associé à chaque type :

$\mathrm{I}_{\mathrm{S}}=\left(\sum \mathrm{EA}_{\mathrm{i}} \times \mathrm{SAU}_{\mathrm{i}} \times \mathrm{I}_{\mathrm{Ti}}\right) /\left(\sum \mathrm{EA}_{\mathrm{i}} \times \mathrm{SAU}_{\mathrm{i}}\right)$

avec : $\mathrm{EA}_{\mathrm{i}}$ le nombre d'exploitations du type $\mathrm{i}$

$\mathrm{SAU}_{\mathrm{i}}$ la SAU moyenne du type $\mathrm{i}$

$\mathrm{I}_{\mathrm{Ti}}$ l'indicateur de risque du type $\mathrm{i}$

Comme l'indicateur de risque $\mathrm{I}_{\mathrm{T}}$, cet indicateur ne doit pas être pris en absolu (par exemple, sa comparaison brute avec le seuil de potabilité en nitrates n'aurait que peu de sens), mais il permet de hiérarchiser les SAGE les uns par rapport aux autres en fonction $\mathrm{du}$ risque de pollution nitrique qui leur est associé.

Les résultats montrent que ce nouvel indicateur présente dans les quatre SAGE des valeurs très proches, non significativement différentes, que le calcul soit fait à partir des lixiviations nitriques moyennes sur sept ans ou sur une année sèche (tableau 3 ).
Cependant, étant calculé à l'échelle des SAGE, cet indicateur cache l'hétérogénéité de ces unités territoriales. En effet, les types d'exploitation présentant les plus forts risques de pollution par les nitrates ne sont pas répartis de façon uniforme sur l'ensemble des SAGE, mais sont concentrés sur plusieurs groupes de communes. Il en est de même pour les types aux indicateurs de risque les plus faibles. Pour mettre en évidence cette hétérogénéité spatiale, le même indicateur de risque a été calculé à l'échelle des communes (figure 4). Il est à noter que, pour le moment, les surfaces forestières - qui sont stables et sous lesquelles les pertes nitriques sont minimes n'ont pas été prises en compte dans le calcul des indicateurs de risque par commune et par SAGE ; ceux-ci s'en trouvent par conséquent surestimés.

Une représentation cartographique de cet indicateur permet de visualiser à l'intérieur de chaque SAGE, les zones susceptibles de présenter des problèmes de pollution nitrique des eaux souterraines (figure 5). Le territoire couvert par la plaine des Vosges dans le SAGE de la Haute-Meuse est un bon exemple de l'hétérogénéité qui peut exister : une partie de ce territoire, qui regroupe une dizaine de communes sur les plateaux de Neufchâteau, présente le plus haut risque de pollution nitrique des eaux, tandis que l'autre partie est constituée d'une majorité de communes, dans la petite région du Bassigny-Châtenois, qui présentent le plus faible risque. Les actions à mettre en œuvre pour protéger les ressources en eau souterraines dans ces deux zones d'un même SAGE ne devront donc pas être identiques. L'hétérogénéité des communes vis-à-vis du risque de pollution

Figure 4. Fréquence des communes par classe d'indicateur de risque.

Nombre de communes

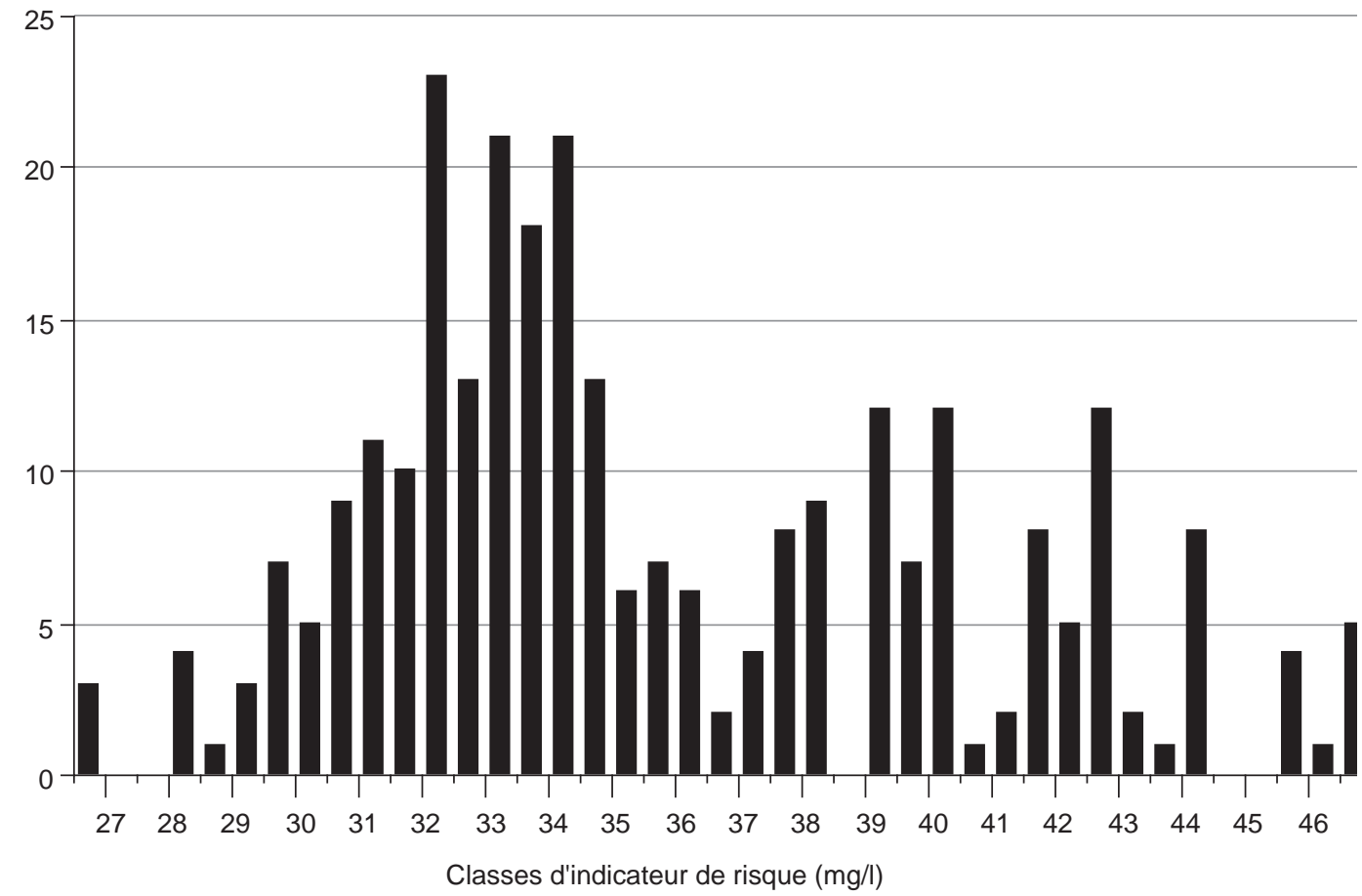


Figure 5. Indicateur des risques de pollution par les nitrates dans les quatre SAGE de la Plaine des Vosges.

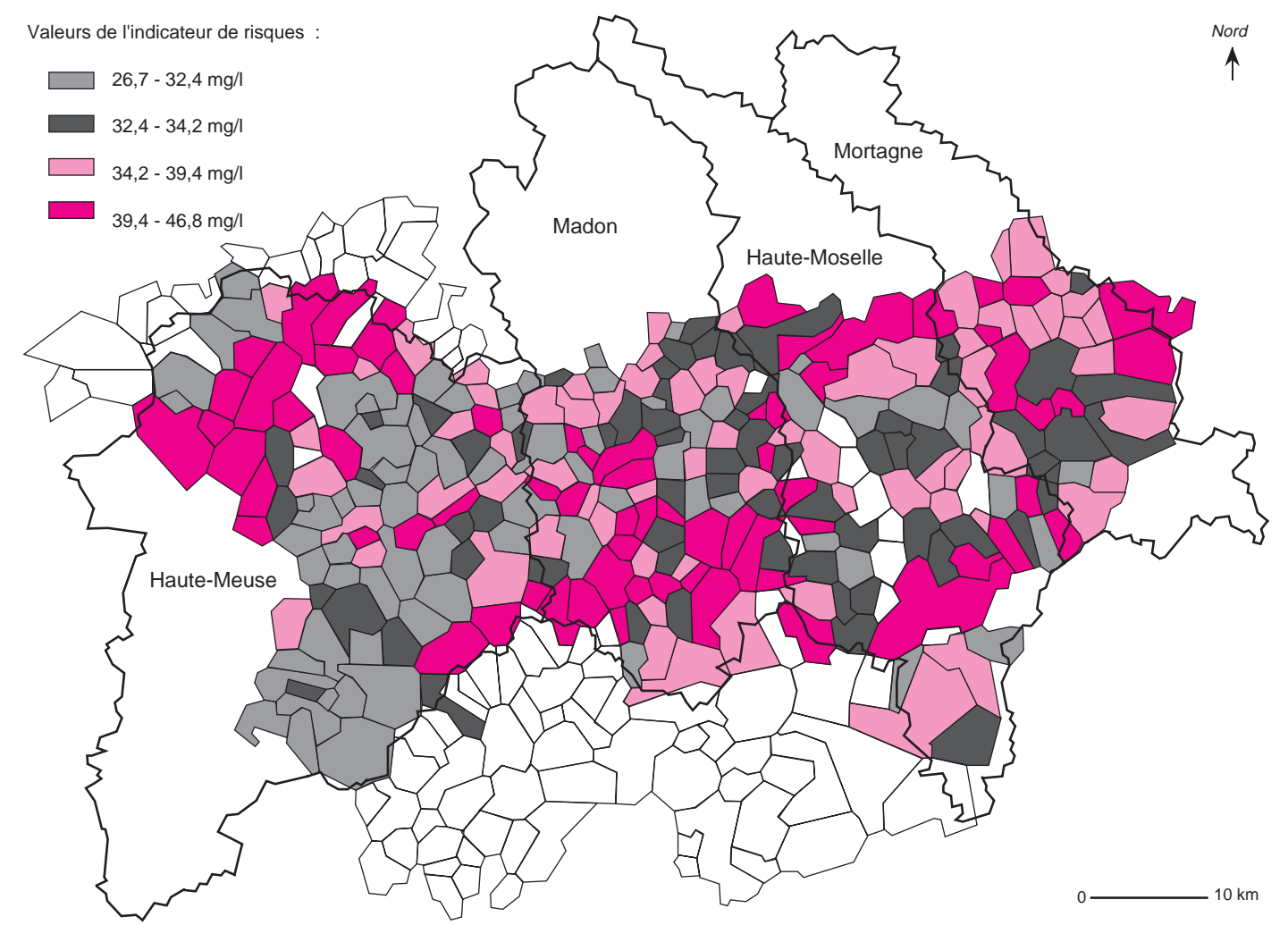

nitrique est également importante dans les trois autres SAGE. Il est à noter que l'on retrouve bien, dans les communes soumises au plus fort risque, celles qui sont caractérisées par les types d'exploitation lait-céréales ou viande-céréales les plus modernisés et intensifs et, dans les communes soumises au plus faible risque, celles qui sont caractérisées par les types les plus herbagers et extensifs.

\section{3 / Les jeux d'échelles spatiales}

Une question méthodologique majeure posée par la démarche présentée est la généralisation des résultats de lixiviations nitriques obtenus à la parcelle à l'ensemble des types d'exploitation et des communes de la plaine des Vosges, même si elle respecte le domaine de validité des mesures. La forte variabilité des pertes nitriques moyennes à l'échelle de la sole (de $17 \mathrm{mg} / \mathrm{l}$ pour les prés de fauche à $93 \mathrm{mg} / \mathrm{l}$ pour le colza) diminue considérablement aux échelles du type d'exploitation et de la commune, et encore plus à l'échelle du SAGE (figure 6). Nous observons ainsi un fort lissage de la dispersion des résultats quand on passe à des niveaux d'agrégation croissants.

Ce constat nous conduit actuellement à augmenter le nombre de parcelles agricoles suivies, de manière à augmenter la représentativité des sites de mesure, en nous basant sur une typologie des systèmes de culture lorrains établie par Regnard (1995). Nous souhaitons ainsi initier un réseau régional de suivi de la qualité de l'eau, qui permettra entre autres d'affiner les indicateurs de risque.
L'échelle du bassin d'alimentation, dont de nombreux travaux d'agronomes ont démontré la pertinence par rapport aux problèmes de qualité des ressources en eau (Hénin et al 1980, Concaret et al 1984, Pierre 1987, Mary et al 1996) est absente de la démarche. Les bassins d'alimentation sont des zones d'infiltration et/ou de ruissellement préférentiel de l'eau qui alimentent les nappes phréatiques. L'occupation des sols des bassins peut donc être directement reliée à la qualité de l'eau des sources qui y sont situées.

Les exploitations présentent des situations inégales par rapport aux bassins d'alimentation. Certaines possèdent une large part de

Figure 6. Dispersion des quantités moyennes de nitrates lixiviés selon l'échelle spatiale.

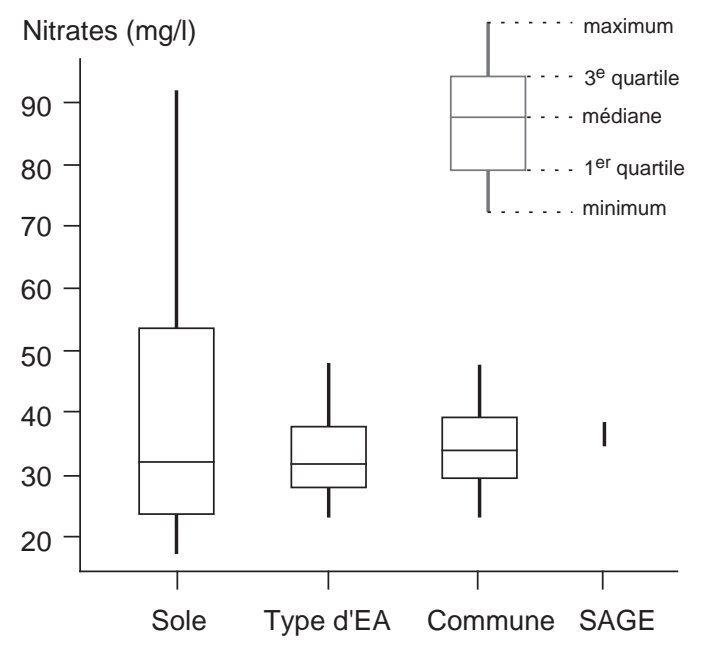

\section{La localisation des types d'exploitation sur la carte des $S A G E$ permet d'identifier les zones où le risque de pollution azotée est le plus élevé.}


leur parcellaire à l'intérieur d'un ou de plusieurs bassins, d'autres au contraire sont situées entièrement hors bassin. Il apparaît ainsi évident que si les premières mettent en œuvre des pratiques agricoles intensives et polluantes, les pertes en nitrates qui en résultent ont beaucoup plus de conséquences sur la qualité de l'eau que les mêmes pertes issues des mêmes pratiques mises en œuvre par les secondes. La pollution par les nitrates liée au fonctionnement des exploitations dépend donc fortement de la localisation de leur parcellaire et des assolements associés par rapport aux bassins d'alimentation (Le Houérou 1993).

Cette échelle spatiale n'est pas intégrée dans notre démarche pour différentes raisons. D'une part, il y a incompatibilité entre la volonté de travailler à grande échelle, sur l'ensemble de la plaine des Vosges, et la prise en compte de l'échelle du bassin ; la délimitation de la totalité des bassins d'alimentation des ressources en eau souterraines situés dans la plaine des Vosges est à l'heure actuelle loin d'être effectuée et, même en disposant de ce découpage, il serait impossible d'y localiser correctement les 2211 exploitations : affecter une exploitation au bassin dans lequel son siège se situe entraînerait un biais inacceptable. D'autre part, mis à part quelques cas particuliers (zone de protection d'une source d'eau minérale, zone de mise en place d'opérations Fertimieux), les bassins d'alimentation correspondent encore trop peu à des niveaux d'organisation au sein desquels des décisions peuvent être prises.

\section{Conclusion}

Ce travail a consisté à élaborer des indicateurs de risque de pollution nitrique diffuse en fonction des types d'exploitation présents dans la région d'étude, et à les spatialiser afin de délimiter des "zones à risque ". Il montre la complémentarité entre des mesures de la lixiviation nitrique réelle sous parcelles, une typologie d'exploitation qui contient des informations sur les assolements, et un système d'information géographique pour situer les résultats au sein d'unités territoriales de gestion de l'environnement. Il n'a pas pour ambition de prédire les teneurs en nitrates précises des sources d'eau potable vosgiennes, mais de contribuer à la mise au point d'outils de diagnostic et d'aide à la décision pour les différents acteurs concernés par la protection des ressources en eau.

Le domaine de validité des mesures et des résultats est actuellement limité aux types de sol et au climat lorrains (hors montagne vosgienne) et aux formes d'activités agricoles présentes dans la zone "plaine ". Dans une étape ultérieure, la démarche et les résultats seront validés en croisant la carte des zones à risque obtenue avec la carte des teneurs en nitrates réelles des sources d'eau potable des Vosges mesurées annuellement par la DDASS.

L'intégration du facteur temps dans la démarche paraît être également une perspective intéressante, surtout dans le contexte actuel de mutation rapide de l'activité agricole. Les dix dernières années ont vu une évolution majeure des structures d'exploitation lorraines, qui a eu des répercussions importantes sur les fonctionnements d'exploitation et, par conséquent, entre autres, sur la qualité des ressources en eau. Il nous semble donc nécessaire de mettre au point des méthodes pour suivre la dynamique des activités agricoles selon deux points de vue :

- la dynamique des systèmes de culture régionaux étudiée par traitement des enquêtes annuelles " utilisation du territoire " réalisées par les Services de statistique agricole (Regnard 1995) et par traitement d'images satellitaires multidates (Benoît et al 1994);

- la dynamique des types d'exploitation que nous projetons de modéliser sous la forme de trajectoires selon une méthode initiée par Perrot et al (1995).

Ces deux éléments, validation des indicateurs et intégration du temps, nous permettront enfin de réaliser des simulations.

\section{Remerciements}

Nous tenons à remercier les personnes qui ont participé à ce travail ou qui nous ont permis d'utiliser leurs données : Damien Foissy et Gilles Rouyer de la Station INRA de Mirecourt, responsables pour le premier du suivi des sites à bougies poreuses et du dépouillement des données et pour le second de l'analyse des solutions du sol en laboratoire ; la Chambre Départementale d'Agriculture des Vosges, qui nous a laissé accéder à certaines informations du Répertoire d'exploitations, et Marie-Noëlle Anfrie qui a assuré la partie cartographique de l'étude.

\section{Références bibliographiques}

Agence de l'Eau Rhin-Meuse, 1995. Schéma directeur d'aménagement et de gestion des eaux, $73 \mathrm{p}+$ annexes. Agence de l'Eau Rhin-Meuse, 57000 Metz.

Barlier J., 1991. Pose et fonctionnement de sites à bougies poreuses en situation agricole. Cahier des techniques de l'INRA, 26, 37-46. INRA Theix, 63122 St Genès Champanelle.

Benoît M., Le Ber F., Bachacou J., 1994. Aerospace surveys and on farm resarch to manage groundwater resources. In : L. Fresco et al (eds), Future of the land, 345-346. Wageningen Agricultural University Press, Pays-Bas.

Benoît M., Saintôt D., Gaury F., 1995a. Mesures en parcelles d'agriculteurs des pertes en nitrates. Variabilité sous divers systèmes de culture et modélisation de la qualité de l'eau d'un bassin d'alimentation. C.R. Acad. Agric., 81, 175-188.

Benoît M., Parrassin P.-R., Peyre D., Fiorelli J.-L., 1995b. Activités d'élevage et qualité des eaux souterraines. Méthodes d'évaluation des risques de pollu- 
tion azotée et d'estimation des pertes en nitrates. Renc. Rech. Ruminants, 2, 323-328.

Concaret J., De Crecy J., Perrey C., 1984. Fonctionnement hydraulique de parcelles drainées en liaison avec la dynamique structurale. Essai d'interprétation. Agronomie, 4, 749-761.

Coppenet, 1975. Bilan des éléments fertilisants sur les exploitations d'élevage. Fourrages, 62, 119-132.

Duthil F., 1973. Eléments d'écologie et d'agronomie. Tome III, 656 p. Editions J.-B. Baillière, Paris.

Fiorelli J.-L., Bazard C., Peyre D., 1995. Rejets azotés par les vaches laitières et lessivage de nitrates en pâturage continu. Renc. Rech. Ruminants, 2, 364 .

Gaury F., 1992a. Systèmes de culture et teneurs en nitrates des eaux souterraines. Dynamique passée et actuelle en région de polyculture-élevage sur le périmètre d'un gîte hydrominéral. Thèse de doctorat de l'ENSA de Rennes, Sciences agronomiques, 229 p + annexes.

Gaury F., 1992b. L'utilisation d'un système d'information géographique pour l'étude des relations entre systèmes de culture et qualité des eaux souterraines. In : P. Buche, D. King, S. Lardon (eds), Gestion de l'espace rural et système d'information géographique, 185-193. INRA, Paris.

Hénin S. et collaborateurs, 1980. Rapport du groupe de travail activités agricoles et qualité des eaux. Ministère de l'Agriculture, Ministère de l'Environnement et du Cadre de Vie, Paris, 34 p + annexes.

Küng-Benoît A., 1992. Réduction de la pollution nitrique : exemple d'un diagnostic en Lorraine. Fourrages, 131, 235-250.

Le Houérou B., 1993. Optimisation des lisiers et fumiers. In : Matières organiques et agriculture. Actes du GEMAS-COMIFER, Blois, France, 16-18 novembre 1993, 103-113.

Machet J.-M., Mary B., 1990. Effet de différentes successions culturales sur les risques de pertes en nitrates en région de grande culture. In : $\mathrm{R}$. Calvet (ed), Nitrates-Agriculture-Eau, Colloque international, Paris-la Défense, 7-8 novembre 1990, 395-403. INRA, Paris.

Mary B., Beaudoin N., Benoît M., 1997. Prévention de la pollution nitrique à l'échelle du bassin d'alimentation en eau. In : G. Lemaire et B. Nicolardot (eds), Maîtrise de l'azote dans les agrosystèmes, Reims (France) 19-20 octobre 1996, Les Colloques $\mathrm{n}^{\circ}$ 83, 289-312. INRA, Paris.

Mignolet C., 1996. Projection spatiale de la diversité des exploitations agricoles du département des Vosges. In : C. Christophe, S. Lardon et P. Monestiez (eds), Etude des Phénomènes Spatiaux en Agriculture, La Rochelle (France), 6-8 décembre 1995. Les Colloques n ${ }^{\circ} 78,143-150$. INRA, Paris.

Mignolet C., Saintôt D., Benoît M., 1996. Livestock farming system diversity and groundwater quality modelling at a regional scale. $4^{\mathrm{e}}$ Symposium international sur les Systèmes d'Elevage en Ferme, Foulum (Danemark), 22-23 août 1996, 6 p.

Perrot C., 1990. Typologie d'exploitations construite par agrégation autour de pôles définis à dire d'experts. INRA Prod. Anim., 3, 51-66.

Perrot C., Pierret P., Landais E., 1995. L'analyse des trajectoires des exploitations agricoles. Une méthode pour actualiser les modèles typologiques et étudier l'évolution de l'agriculture locale. Economie Rurale, 228, 35-47.

Pflimlin A., Madeline Y., 1995. Evaluation des risques de pollution nitriques liés à l'élevage de ruminants et stratégies d'intervention pour la qualité de l'eau. Renc. Rech. Ruminants, 2, 329-338.

Pierre D., 1987. Utilisation agronomique des bassins versants : problèmes méthodologiques et exemples d'application. Perspectives Agricoles, 115, 171-183.

Regnard C., 1995. Constitution d'un observatoire de la qualité des eaux issues des systèmes de culture lorrains à partir de parcelles drainées sélectionnées chez les agriculteurs. Mémoire de fin d'études, ISA de Beauvais, $47 \mathrm{p}+$ annexes.

\section{Abstract}

Livestock farming systems and nitrate pollution risk. Construction of a risk indicator and application on the Vosges plain.

A method for evaluating and localizing areas where there are risks of diffusing nitrates into the groundwater has been established and linked to the livestock farming system diversity and to their characteristic cropping plans. It has been applied to the 2211 farms present in 1993 in the Vosges plain. This method associates real nitrate leaching measures under farm fields, a livestock farm typology which contains information on the cropping plans, and a geographic information system to locate the information within water resource management units. Currently, the farm types combining big areas in cash crops and a maize forage system present the highest potential risks of nitrate pollution, while the farm types specialised in grassland dairy and/or meat production seem to be the least polluing. The farm type location makes it possible to identify geographic areas exposed to a high agricultural nitrate pollution risk, which are characterized by a combination of the most modernised and intensive farm types of the region.

Mignolet C., Benoît M., Saintôt D., 1997. Systèmes d'élevage et risque de pollution azotée. Construction d'un indicateur de risque et application dans la plaine des Vosges. INRA Prod. Anim., 10 (4), 275285. 